\title{
Capsule Commentary on Meddings et al., The Impact of Disability and Social Determinants of Health on Condition-Specific Readmissions Beyond Medicare Risk-Adjustors: A Cohort Study
}

Jessica Meister-Berger, MD, JD

Medical College of Wisconsin, Milwaukee, WI, USA.

$\mathrm{J}$ Gen Intern Med 32(1):91

DOI: $10.1007 / \mathrm{s} 11606-016-3894-9$

(c) Society of General Internal Medicine 2016

$\mathrm{R}$ eadmission rates are influenced by complex patient factors, including comorbidities, age, and gender. ${ }^{1}$ The Centers for Medicare and Medicaid Services (CMS) use a condition-specific risk adjustment model based on this administrative data to determine reimbursement for readmissions. ${ }^{2}$ However, there is growing concern that current risk adjustment models oversimplify and therefore fail to adequately account for disability and social determinants of health, which may influence readmission rates by affecting a patient's ability to comply with post-discharge treatment plans. ${ }^{3-5}$ Thus, under the current model, hospitals are financially penalized for readmissions driven by complex factors beyond their control.

This retrospective cohort study of Medicare patients assessed the predictive strength of disability and select social determinants on readmission. The study examined the effects of multiple factors, including socioeconomic status, race, marital status, activities of daily living (ADL) limitations, and home health care needs, on readmission rates for patients hospitalized for pneumonia, heart failure, and acute myocardial infarction (AMI). Despite the statistical significance of disability and several social determinants of health in individual predictor models, in full models no predictors were statistically significant across all three cohorts. Prior home health care needs and having three or more ADL limitations were the only statistically significant predictors for readmission in patients hospitalized for pneumonia, while in patients treated for AMI, only nursing home residence and race were statistically significant. In the heart failure cohort, non-white race was a significant predictor of readmission, whereas having children and being in the highest quartile of wealth were significant predictors of reduced readmission.
Further studies are warranted in an effort to develop risk adjustment models that more accurately and equitably reflect the multi-factorial impetus behind readmissions. Although logistically formidable, implementation of a more robust risk stratification model would be a timely evolution in the current milieu of health care economics. Moreover, this study subtly highlights an undercurrent of societal apathy inherent in the present model's failure to account for and adequately address implications of socioeconomic and racial factors on readmissions, which in itself portends deep concerns of social injustice that are arguably perpetuated by utilization of a risk adjustment model that ignores these complexities.

Corresponding Author: Jessica Meister-Berger, MD, JD; Medical College of Wisconsin, Milwaukee, WI, USA (e-mail:jberger@mcw.edu).

\section{Compliance with Ethical Standards:}

Conflict of Interest: The author has no conflicts of interest with this article.

\section{REFERENCES}

1. Meddings J, Reichert H, Smith SN, Iwashyna TJ, Langa KM, Hofer TP, McMahon LF. The Impact of Disability and Social Determinants of Health on Condition-Specific Readmissions Beyond Medicare Risk-Adjustors: A Cohort Study. J Gen Intern Med. doi:10.1007/s11606-016-3869-X

2. Yale New Haven Health Services Corporation/Center for Outcomes Research \& Evaluation (YNHHSC/CORE). 2013 Measures Updates and Specifications Report: Hospital-Level 30-Day Risk-Standardized Readmission Measures for Acute Myocardial Infarction, Heart Failure, and Pneumonia (Version 6.0). 2013. Available at https://www.qualitynet.org/dcs/ContentServer? cid $=1228774371008 \&$ pagename $=$ QnetPublic $\% 2 \mathrm{FPage} \% 2 \mathrm{FQnetTier} 4 \& \mathrm{c}=\mathrm{Pa}-$ ge. Accessed September 21, 2016.

3. Barnett ML, Hsu J, McWilliams JM. Patient characteristics and differences in hospital readmission rates. JAMA Intern Med. 2015;175(11):1803-12.

4. Joynt KE, Jha AK. Thirty-day readmissions-truth and consequences. N Engl J Med. 2012;366(15):1366-9.

5. Kansagara D, Englander H, Salanitro A, et al. Risk prediction models for hospital readmission: a systematic review. JAMA. 2011;306(15):1688-98.

Published online October 11, 2016 